\title{
PATRONES DE NIDIFICACIÓN DE Trigona (Tetragonisca) angustula Y Melipona rufiventris (Hymenoptera: Meliponini) EN EL NORTE DE LA PAZ, BOLIVIA
}

\section{NESTING PATTERNS OF Trigona (Tetragonisca) angustula Y Melipona rufiventris (Hymenoptera: Meliponini) IN NORTHERN LA PAZ, BOLIVIA}

\author{
María Copa - Alvaro ${ }^{1}$
}

Tres comunidades Tacanas en el norte de La Paz, con el objeto de criar abejas nativas, trasladaron colonias silvestres de su bosque aledaño a cajas racionales. De estas colonias se registró información sobre: tipo de bosque y especies de árboles donde anidan, diámetro a la altura del pecho del árbol hospedero y altura al tubo de ingreso. Trigona (Tetragosnisca) angustula $(\mathrm{n}=$ 100) ubica sus nidos principalmente en áreas de barbecho (descanso de cultivos, chacos y potreros) con mayor frecuencia en la especie arbórea Astronium urundeuva; la otra especie de abeja Melipona rufiventris $(\mathrm{n}=24)$ ubicó sus nidos en la matriz boscosa y no se encontraron señales de preferencia por alguna especie arbórea. El mayor porcentaje de nidos de T. angustula fue encontrado en árboles muertos y la altura promedio al tubo de ingreso fue registrado en $32 \mathrm{~cm}$ (DS =24.4); el diámetro promedio a la altura del pecho de los árboles hospederos fue de $37 \mathrm{~cm}$ (DS = 13.4) mientras que para $M$. rufiventris fue de $42 \mathrm{~cm}(\mathrm{DS}=14.5)$. Este trabajo es el primer estudio de nidificación de estas abejas en la zona y en Bolivia

Palabras clave: abejas sin aguijón, nido, especies de árboles, hábitat, Bolivia.

\begin{abstract}
Three Tacana communities in northern La Paz wanted to moved wild colonies from a nearby forest to beehives with the purpose of raising native bees. Previously, we registered data on the type of forest and the species of trees where they built their nests, the diameter at breast height (dbh) of host trees, and the height of the entrance tube. Trigona (Tetragosnisca) angustula $(\mathrm{n}=$ 100) had their nets principally in disturbed forest areas (agricultural field, abandoned fields, chacos and horse pastures), and with higher frequency in the tree species Astronium urundeuva; whereas Melipona rufiventris $(\mathrm{n}=24)$ located their nests within the forest and we did not encounter signs of preference for any tree species. The highest percentage of $T$. angustula nests were found in dead trees, and the mean height of the entrance tube was $32 \mathrm{~cm}(\mathrm{DS}=24.4)$. The average diameter at breast height for its host trees was $37 \mathrm{~cm}(\mathrm{DS}=13.4)$, while it was $42 \mathrm{~cm}(\mathrm{DS}=14.5)$ for $M$. rufiventris.
\end{abstract}

Key words: stingless bees, nests, tree species, habitat, Bolivia.

\section{Introducción}

Dentro de la tribu Meliponini, que agrupa a todas las abejas sin aguijón, se encuentran dos géneros comunes y de importancia económica en las tierras bajas de Sudamérica: Trigona y Melipona.

Además del interés biológico por este grupo, los ingresos generados por el comercio de miel, cera y otros productos, han impulsado la búsqueda de información local sobre biología y manejo racional de estas especies en el rango de su distribución.

En general, nidifican casi en cualquier cavidad que encuentren disponible, agujeros de árboles, pisos, paredes, nidos abandonados de ceramícidos o nidos vivos de termitas y hormigas. En Costa Rica se observó en un área de ecotono bosque - sabana que los nidos del género Trigona se ubicaban entre 60 y $180 \mathrm{~m}$ al borde (Hubbell \& Jhonson, 1977). Cuando los nidos no son subterráneos las alturas a las cuales nidifican oscilan entre el nivel del suelo hasta los $12 \mathrm{~m}$ (Nates-Parra, 1996). La altura a la piquera o tubo de ingreso encontrada para Melipona favosa fue $22 \pm 0.5$ m (Moreno \& Cardozo, 2002)

En el Estado de Portuguesa - Venezuela se ha observado que en condiciones naturales el árbol más utilizado por Melipona compressipes y Scaptotrigona $s p$. es la especie maderable Pithecellobium saman (Leg. Mimosoideae); Trigona (Tetragonisca) angustula utiliza los troncos de los árboles de Astronium graveoleus (Anacardiaceae) y Trichanthera gigantea (Acantaceae) (Moreno \& Cardozo, 2002). Sin embargo, existen lugares donde no se ha observado ninguna preferencia por el uso de especies arbóreas como Costa Rica (Hubbell \& Johnson, 1977).

Algunas especies, como Apis mellifera, Nannotrigona testaceicornis y Trigona (Tetragonisca) angustula, se adaptan muy bien a las condiciones urbanas, siendo bastante comunes en las ciudades de Brasil (Moreno \& Cardozo, 1997; Silva \& Amaral, 1996). 
En Bolivia, aunque son pocos los que trabajan con abejas nativas, principalmente grupos originarios, el interés aumenta con el tiempo. Un ejemplo claro son tres comunidades Tacanas que se unieron para solicitar apoyo en la iniciación de la meliponicultura en su zona.

Con esta iniciativa y la necesidad de conocer los requerimientos locales de las abejas en cuanto a nidificación, se tomaron datos sobre diámetro a la altura del pecho, altura de los árboles hospederos y altura al tubo de ingreso de los nidos. Con el propósito de ganar un mejor entendimiento sobre las preferencias de estas abejas para establecer sus nidos, nos planteamos las siguientes preguntas: 1. ¿cuáles son las características del bosque utilizado con más frecuencia por las dos especies?, 2. ¿qué árboles fueron utilizados en mayor frecuencia?.
W $68^{\circ} 06^{\prime} 28^{\prime \prime}$ ') y Carmen Pecha (S $13^{\circ} 45^{\prime} 55^{\prime \prime}$ y W $68^{\circ} 04^{\prime} 53^{\prime}$ '), se encuentran en el municipio de Ixiamas y pertenecen a la Tierra Comunitaria de Origen Tacana, en el Norte del Departamento de La Paz (Figura 1).

La temperatura promedio en la zona es $26^{\circ} \mathrm{C}$ con $1800-2000 \mathrm{~mm}$ de precipitación anual, la época de lluvias se prolonga de diciembre a marzo y la seca de junio a octubre (CIPTA - WCS, 2002).

Santa Fe presenta fragmentos de sabana arbolada rodeada por bosque húmedo amazónico, la comunidad se encuentra a $7 \mathrm{~km}$ de la base de la Serranía El Tigre. En el área de San Pedro domina la sabana arbolada, el bosque de donde se obtuvieron las abejas, es un bosque de galería, formación ubicada alrededor de los arroyos principales que se prolongan hasta su desembocadura en el río Beni. Carmen Pecha al igual

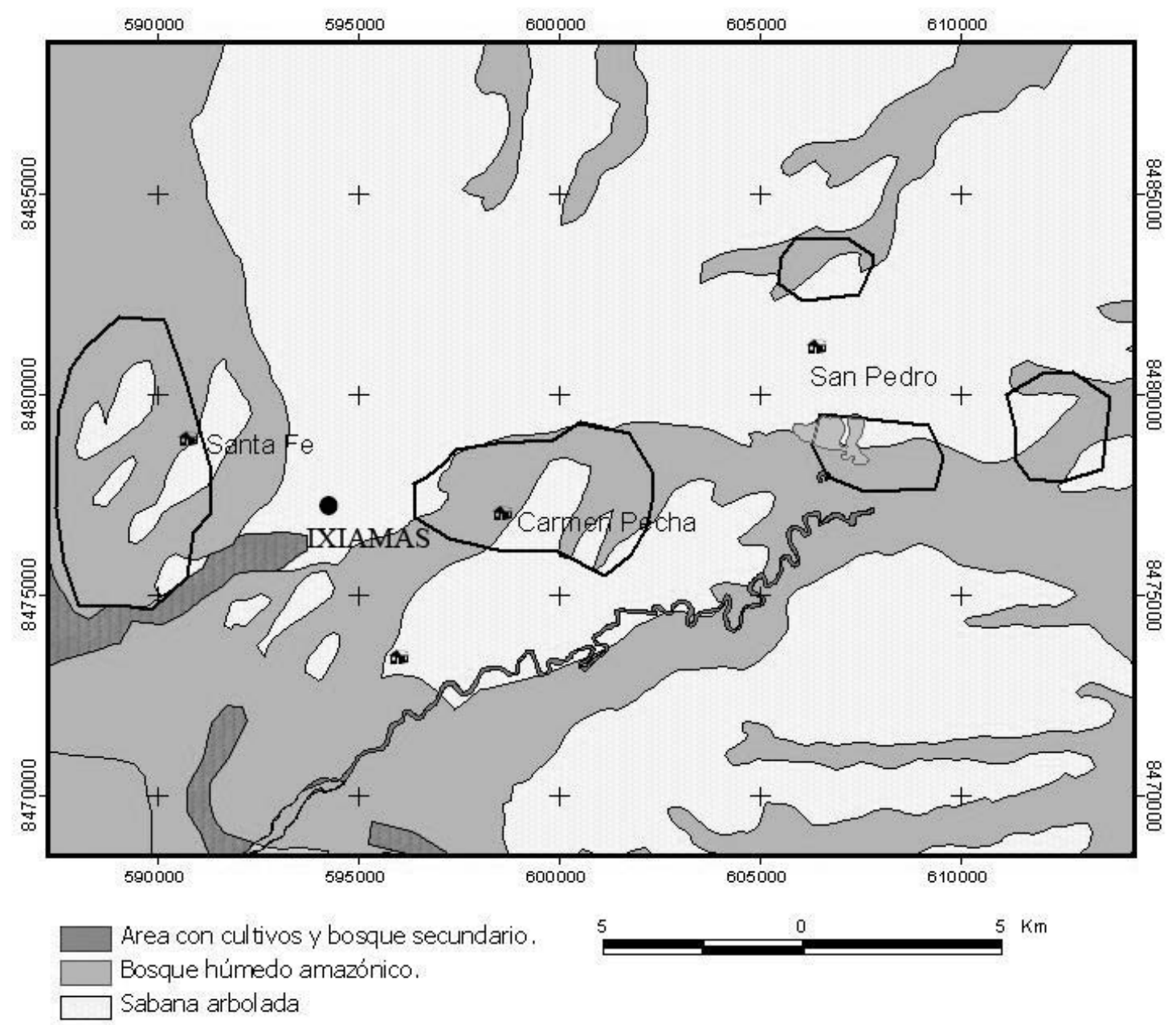

Figura 1. Área de estudio.

\section{Materiales y métodos \\ Área de estudio}

Las tres comunidades Tacanas; Santa Fe (S $13^{\circ}$ 44' 58', W 68 09' 22''), San Pedro (S 13 $3^{\circ} 48^{\prime} 05^{\prime}$ ', que San Pedro se encuentra en una zona con sabanas arboladas y el bosque en galería de donde provienen las abejas es el mismo de San Pedro (Figura 1).

La principal actividad de los comunarios es la agricultura. Anualmente limpian áreas boscosas para utilizarlas como nuevas tierras de cultivo, 
paralelamente dejan una superficie similar como área de recuperación en nutrientes denominada barbecho (CIPTA - WCS, 2002).

\section{Mediciones en los sitios de nidificación}

Meses previos al traslado, los comunarios en sus recorridos cotidianos a las áreas de chaco, salidas de cacería y caminatas al bosque, buscaron nidos de abejas nativas sobre las sendas ya establecidas, no abrieron nuevas sendas. La búsqueda fue principalmente hasta donde la vista les permitía distinguir los tubos de ingreso y las abejas sobrevolando el mismo, en algunos casos ubicaron nidos por el zumbido revelador en caminatas nocturnas. Únicamente en la comunidad de San Pedro se realizó una salida específica para encontrar la mayor cantidad de nidos, el lugar que tenia una cantidad importante de nidos para el traslado es una zona que antiguamente era transitada por sus antepasados (J. Dapara com. pers., 2003).

En Junio 2001 se realizó el traslado de las primeras colonias silvestres a cajas racionales, por cada una de las colonias trasladas se tomaron los siguientes datos: especie de abeja, hábitat (diferenciado en barbecho, ecotono bosque - sabana y bosque), especie arbórea, diámetro a la altura del pecho (DAP), diámetro interno y altura en la que se encontró el ingreso al nido. Todos los árboles fueron diferenciados en dos categorías: árboles vivos y troncos (árboles muertos caídos o parados).

Después de un primer traslado conjunto entre personal técnico y comunarios, la comunidad se hizo cargo de los demás traslados, por esta razón muchos datos acordados no fueron tomados y entonces el tamaño de muestra para cada uno de los parámetros no es el mismo. También debemos resaltar que los registros corresponden a nidos encontrados a alturas menores a metro y medio, esto debido a la dificultad que se presenta en el momento de los traslados, sacar el nido sin causarle daño.

El proyecto otorgó un total de 150 cajas 125 para $T$. angustula y 25 para $M$. rufiventris.

Para estimar la cantidad de árboles caídos en los alrededores se realizó una medición de densidad de árboles; en un transecto de $200 \mathrm{~m}$ de longitud, cada 20 m se ubicó una cruz a partir del cual se tomó las distancias a los árboles vivos y a los troncos más cercanos. Con estas distancias se obtuvo la densidad como lo muestra Rabinowitz (2003).

Las medidas son expresadas en promedio \pm DS, para determinar diferencias entre especies en cuanto a la altura al tubo de ingreso y el diámetro interno del árbol hospedero se utilizó una prueba t-student (two tailed). Se utilizó una prueba de Chi - cuadrado para determinar si el encuentro de los nidos fue diferente en algún tipo de hábitat, el valor esperado fue igual para todos los hábitats ya que inicialmente no existió sesgo en la búsqueda de nidos, nivel de significancia 0.05 .

\section{Resultados}

En total se trasladaron 130 colonias de abejas silvestres, de las cuales 100 fueron de T. angustula (19 en Carmen Pecha, 48 en Santa Fe y 33 en San Pedro), 24 de M. rufiventris (6 en Carmen Pecha, 10 en Santa Fe y 8 en San Pedro) y el resto otras especies.

En la Tabla 1 se encuentra el resumen de los patrones observados en la nidificación de ambos tipos de abejas.

Tabla 1. Resumen de los patrones encontrados en los nidos de Trigona (Tetragonisca) angustula y Melipona rufiventris.

\begin{tabular}{|l|l|c|l|l|}
\hline Parámetros & T. angustula & N & $\begin{array}{l}\text { M. } \\
\text { rufiventris }\end{array}$ & N \\
\hline \multirow{2}{*}{ Hábitat } & Barbecho * & 45 & Barbecho & 4 \\
\cline { 2 - 5 } & Ecotono & 22 & Ecotono & 4 \\
\cline { 2 - 5 } & Bosque * & 18 & Bosque * & 12 \\
\hline $\begin{array}{l}\text { Especie hospedera } \\
\text { frecuente }\end{array}$ & $\begin{array}{l}\text { Astronium } \\
\text { urundeuva }(47 \%)\end{array}$ & 85 & - & 14 \\
\hline $\begin{array}{l}\text { Diámetro a la altura del } \\
\text { pecho }\end{array}$ & $37.7 \pm 13.4$ & 71 & $42.4 \pm 14.5$ & 14 \\
\hline Altura de árboles vivos & $15.9 \pm 8.9$ & 30 & $19.4 \pm 6.5$ & 11 \\
\hline $\begin{array}{l}\text { Altura al pico de } \\
\text { ingreso ** }\end{array}$ & $32.2 \pm 24.4$ & 75 & $97.2 \pm 116.9$ & 18 \\
\hline Diámetro interno ** & $10.2 \pm 2.87$ & 7 & $17.5 \pm 2.76$ & 3 \\
\hline
\end{tabular}

* Prueba $\mathrm{Chi}^{2}$; por especie: $\mathrm{P}<0.05$.

** Prueba t-student (2-tailed) entre especies: $\mathrm{P}<0.05$

\section{Trigona (Tetragonisca) angustula o señorita}

Hábitat: Los nidos en barbecho (que incluye chacos, potreros y áreas abiertas) fueron encontrados en una frecuencia mayor a la esperada $\left(\mathrm{Chi}^{2}=9.8 ; \mathrm{gl}=1 ; \mathrm{P}<\right.$ 0.01 ), el número de nidos en el ecotono bosque sabana no fue diferente a lo esperado y en la matriz boscosa ligeramente menor $\left(\mathrm{Chi}^{2}=3.8 ; \mathrm{gl}=1 ; \mathrm{P}=\right.$ 0.05).

Especies arbóreas y características de la entrada: 24 especies arbóreas fueron utilizadas por las abejas sin aguijón para establecer los nidos (Tabla 2), Astronium urundeuva (Anacardiaceae) fue utilizada en un $47 \%$. Cuarenta y dos por ciento de los nidos se encontraron en troncos de árboles secos o caídos. La altura promedio de los árboles vivos fue $15.89 \mathrm{~m}(\mathrm{DS}=8.9)$ y el diámetro de la cavidad interna fue $10.2 \mathrm{~cm}(\mathrm{DS}=$ 2.87).

La frecuencia de nidos ubicados en árboles y troncos de $A$. urundeuva (47 \%) fue mayor a la esperada (14\%), basados en la densidad de árboles de esta especie $\left(\mathrm{Chi}^{2}, \mathrm{P}<0.01, \mathrm{gl}=1\right)$

\section{Melipona rufiventris o Erereu}

Hábitat: El hábitat en que se encontró mayor cantidad de colonias es bosque húmedo amazónico, la frecuencia de encuentros fue mayor a la esperada $\left(\mathrm{Chi}^{2}=4.3 ; \mathrm{gl}=1 ; \mathrm{P}<0.05\right)$.

Especies arbóreas e ingreso: 17 especies arbóreas fueron utilizadas para ubicar los nidos $(\mathrm{n}=20) . M$. 
rufiventris no mostró preferencia por ninguna de los árboles en particular (Tabla 2); $17 \%$ de los nidos se encontraron en troncos o árboles caídos; la altura promedio de los árboles vivos fue de $19.4 \mathrm{~m}$ (DS = 6.5). Seis nidos fueron ubicados en la base de árboles con aletones y el diámetro interno donde se encontraron los nidos fue 17.5 ( $\mathrm{DS}=2.76$ ).

Tabla 2. Frecuencia de nidos encontrados en especies arbóreas. Trigona (Tetragonisca) angustula, $\mathrm{N}=100 ;$ Melipona rufiventris, $\mathrm{N}=24$.

\begin{tabular}{|c|c|c|c|}
\hline & 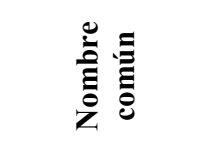 & 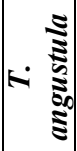 & ฉ \\
\hline \multicolumn{4}{|l|}{ Familia Verbenaceae } \\
\hline Vitex pseudolea & taruma & 0.01 & \\
\hline \multicolumn{4}{|l|}{ Familia Ulmaceae } \\
\hline Phyllostylon sp. & cuta & 0.01 & 0.08 \\
\hline \multicolumn{4}{|l|}{ Familia Tiliaceae } \\
\hline Apeiba membranaceae & cabeza de mono & & 0.04 \\
\hline \multicolumn{4}{|l|}{ Familia Phytolacaceae } \\
\hline Gallesia integrifolia & ajo ajo & 0.01 & \\
\hline \multicolumn{4}{|l|}{ Familia Moraceae } \\
\hline Ficus sp. & bibosi & & 0.04 \\
\hline Poulsenia armata & chamane & 0.03 & 0.04 \\
\hline Bastacarpus costaricensis & chicle, mascajo & & 0.04 \\
\hline Ficus sp. & mata palo & 0.04 & 0.04 \\
\hline Claricia racemosa & mururé & 0.01 & \\
\hline Pseudolmedia laevis & nui & 0.03 & 0.08 \\
\hline \multicolumn{4}{|l|}{ Familia Meliaceae } \\
\hline Trichilia sp. & shapuraqui & 0.01 & \\
\hline \multicolumn{4}{|l|}{ Familia Guttiferae } \\
\hline Clophyllum brasiliensis & palo María & 0.01 & \\
\hline \multicolumn{4}{|l|}{ Familia Fabaceae } \\
\hline Miroxylon balsamun & recino & 0.02 & 0.04 \\
\hline \multicolumn{4}{|l|}{ Familia Euphorbiaceae } \\
\hline Sapium sp. & leche leche & & 0.04 \\
\hline \multicolumn{4}{|l|}{ Familia Combretaceae } \\
\hline Terminalia sp. & verdolago & 0.03 & 0.04 \\
\hline Terminalia sp. & verdolago blanco & 0.01 & \\
\hline Terminalia $s p$. & $\begin{array}{c}\text { verdolago de } \\
\text { pampa }\end{array}$ & 0.01 & \\
\hline \multicolumn{4}{|l|}{ Familia Cecropiaceae } \\
\hline Cecropia sp. & ambaibo & & 0.04 \\
\hline \multicolumn{4}{|l|}{ Familia Bombacaceae } \\
\hline Ochroma pyramidale & balsa & 0.01 & \\
\hline \multicolumn{4}{|l|}{ Familia Bignoniaceae } \\
\hline \multirow[t]{2}{*}{ Tabebuia serratifolia } & tajibo & 0.03 & \\
\hline & tajibo macho & 0.01 & \\
\hline \multicolumn{4}{|l|}{ Anacardiaceae } \\
\hline Spondias monbin & cedrillo & & 0.04 \\
\hline Astronium urundeuva & cuchi & 0.47 & 0.04 \\
\hline sin identificar & cari cari & 0.01 & \\
\hline sin identificar & chaquillo & 0.02 & 0.08 \\
\hline sin identificar & cuatidire & & 0.04 \\
\hline sin identificar & curupaú & & 0.04 \\
\hline
\end{tabular}

\begin{tabular}{|l|c|c|c|}
\hline sin identificar & manicillo & 0.02 & \\
\hline sin identificar & nandú & & 0.04 \\
\hline sin identificar & quina quina & 0.01 & \\
\hline sin identificar & sumaqui & 0.01 & \\
\hline $\sin$ identificar & toco & 0.02 & \\
\hline & $\mathrm{sp}$ & 0.15 & 0.18 \\
\hline
\end{tabular}

\section{Densidad de árboles - troncos}

Se registró una densidad de 0.17 árboles vivos $/ \mathrm{m}^{2}$ y 0.08 troncos $/ \mathrm{m}^{2}$. De los árboles vivos las especies más comunes fueron: Inga $s p$. (14\%) y una especie no identificada denominada localmente como jachao (20 $\%)$; en la categoría de troncos, árboles muertos, la especie más abundante fue también Inga $s p$. (18\%) seguida por Cecropia sp. (14\%) y A. urundeuva $(14 \%)$.

\section{Discusión}

Las características de los nidos encontrados en Ixiamas indican que $T$. angustula ubica sus nidos generalmente en barbechos, áreas utilizadas en la agricultura que permanecen en descanso por algunos años; es posible que la mayor ocurrencia se deba a la mayor variedad y abundancia de flores que las abejas pueden encontrar en este tipo de bosque, un área en descanso generalmente tiene una cantidad importante de especies pioneras, hiervas, lianas y pequeños arbustos. Además de esto la cantidad de cavidades adecuadas puede también ser un factor importante en la selección de un hábitat por estas abejas.

Las actividades humanas reflejadas en los cultivos, barbechos, áreas de extracción maderera y otros, han generado una mayor disponibilidad de árboles caídos y pedazos de troncos que son utilizados por las abejas señoritas. Sin embargo, estas cavidades efímeras mueven a las abejas a buscar otra cavidad en un tiempo más corto que estando en un bosque natural donde los árboles vivos ofrecen cavidades seguras a largo plazo y entonces un menor esfuerzo dedicado a la búsqueda y el establecimiento de nuevos nidos.

Según Hubbell \& Johnson (1977), el uso de cavidades no depende de las especies arbóreas sino de la disponibilidad de cavidades; es posible que la preferencia por A. urundeuva (Anacardiaceae) observada en la zona de Ixiamas, se deba en parte a la alta frecuencia de cavidades que esta especie puede ofrecer, pero además de esto esta especie tiene otras características favorables para las abejas, como ser dureza y resistencia, por lo cual es utilizado en la construcción de viviendas, así, la oferta de un refugio adecuado por el difícil acceso a los depredadores puede aumentar la frecuencia de encuentros en este árbol. Otra propiedad importante del género Urundeuva es que resulta fuertemente aromática, característica que podría ser importante en el momento de la prospección por las abejas para instalar nuevas cavidades, aumentando así su uso. 
Pese a que la búsqueda de nidos fue únicamente hasta metro y medio observamos que $T$. angustula ubica sus nidos por debajo de los $50 \mathrm{~cm}$, es probable que la altura promedio para M. rufiventris, de $97 \mathrm{~cm}$, no sea representativo ya que la búsqueda no se realizó a mayores alturas y según Moreno \& Cardoso (2002) puede ubicar sus nidos a alturas mayores a $22 \mathrm{~m}$. Las menores alturas de ingreso en T. angustula pueden ser un producto de la preferencia de ubicación en trocos, árboles muertos que generalmente están caídos así sus piqueras de ingreso también se encontraran a este nivel.

Por el rango de vuelo no superior a los $2 \mathrm{~km}$ (Nates-Parra, 1996) y por la baja frecuencia en la formación de enjambres (Roubick, 1989), $M$. rufiventris encuentra en la matriz boscosa del bosque húmedo amazónico, cavidades resistentes en árboles vivos, altos y gruesos para ubicar sus nidos, probablemente porque los recursos utilizados se encuentran en el dosel. Esta característica haría susceptible a la especie frente a cambios estructurales en el bosque.

\section{Conclusiones}

Es importante que en el inicio y a lo largo del manejo en la crianza de abejas nativas se considere las características de los lugares de nidificación en condiciones naturales, T. angustula ubica sus nidos principalmente en troncos de $A$. urundeuva, para $M$. rufiventris no se observaron preferencias, sin embargo, los árboles utilizados por esta abeja tienen un DAP promedio de $42 \mathrm{~cm}$ y una altura de $19 \mathrm{~m}$, árboles grandes que generalmente se encuentran en el bosque y no en barbechos donde se observó mayor ocurrencia de nidos de abejas señorita (T. angustula).

\section{Agradecimientos}

A todos los comunarios que independientemente realizaron los registros, a Julio Dapara y Antenor Dapara por proporcionarme información importante de la comunidad de San Pedro y a todos los participantes de la crianza de abejas nativas por su esmero, participación y amistad genuina. A Juan Clemente por su instrucción inicial sin la cual no hubiera sido posible iniciar el traslado. A Teresa Tarifa por el apoyo en el manuscrito. El presente trabajo forma parte del programa de Automonitoreo realizado por las Comunidades Tacanas para alcanzar la participación de los pobladores en el manejo de los recursos en su área de alcance, con apoyo del Programa "Conservación de Biodiversidad a Nivel Paisaje" de la Wildlife Conservation Society financiado por USAID/ Global a través del Acuerdo Cooperativo LAG-A-00-99-00047-00. Las opiniones aquí expresadas representan a los autores y no necesariamente reflejan los criterios de USAID.

\section{Literatura citada}

CIPTA - WCS. 2002. Estrategia de desarrollo sostenible de la Tierra Comunitaria de Origen Tacana con base en el manejo de los recursos naturales 2001 - 2005. WCS - Bolivia.

Hubbell S.P. \& Johnson L.K. 1997. Competition and nest spacing in a tropical stingless bee community. Ecology. 58: 949-963.

Moreno F.A. \& Cardozo A.F. 1997. Abundancia de abejas sin aguijón (Meliponinae) en especies maderables del estado de Portuguesa, Venezuela. Vida Silvestre Neotropical. 6 (1-2): 53-56.

Moreno F.A. \& Cardozo A.F. 2002. Técnicas de campo para localizar y reconocer abejas sin aguijón (Meliponinae).

Nates-Parra G. 1996. Abejas sin aguijón (Hymenoptera: Meliponini) de Colombia. En: Insectos de Colombia. Academia Javeriano. Bogota.

Rabinowitz A. 2003. Manual de capacitación para la investigación de campo y la conservación de la vida silvestre. Wildlife Conservation Society.

Roubick D. 1989. Ecology and natural history of tropical bees. Cambridge. University Press, Cambridge, 514 pp. En: Martins, C. F., CortopassiLaurino, M., Koedam, D. \& V. L. ImperatrizFonseca. (s/f). The use of tree for nesting by stingless bees in Brazilian Caatinga.

Silva R. \& Amaral F. 1996. Influencia de um gradiente de rbanizacao na abundancia, riqueza e composicao em espécies de abelhas em Belo Horizonte (MG). XXII Congresso de Brasileiro de Zoología.

\footnotetext{
${ }^{1}$ Wildlife Conservation Society - Living Landscape Program, Northwestern Bolivian Andes Landscape Project, Casilla 3 - 35181 San Miguel, La Paz - Bolivia

Dirección actual: Alto Obrajes, Sector “A”, Calle Félix Eguino No 483, Tel. 2-731027, La Paz - Bolivia. Correo electrónicomecopa@hotmail.com
} 